\title{
Avaliação da espessura da camada de fibras nervosas da retina e mácula em pacientes com ambliopia
}

\author{
Thickness of the retinal nerve fiber layer, \\ macular thickness, in patients with amblyopia
}

Juliana Mitreㄹ, Ana Laura de Araújo Moura², Halim Fares Neto³, Renata Ramos Verri ${ }^{4}$, Jose Ricardo Carvalho Lima Rehder ${ }^{5}$

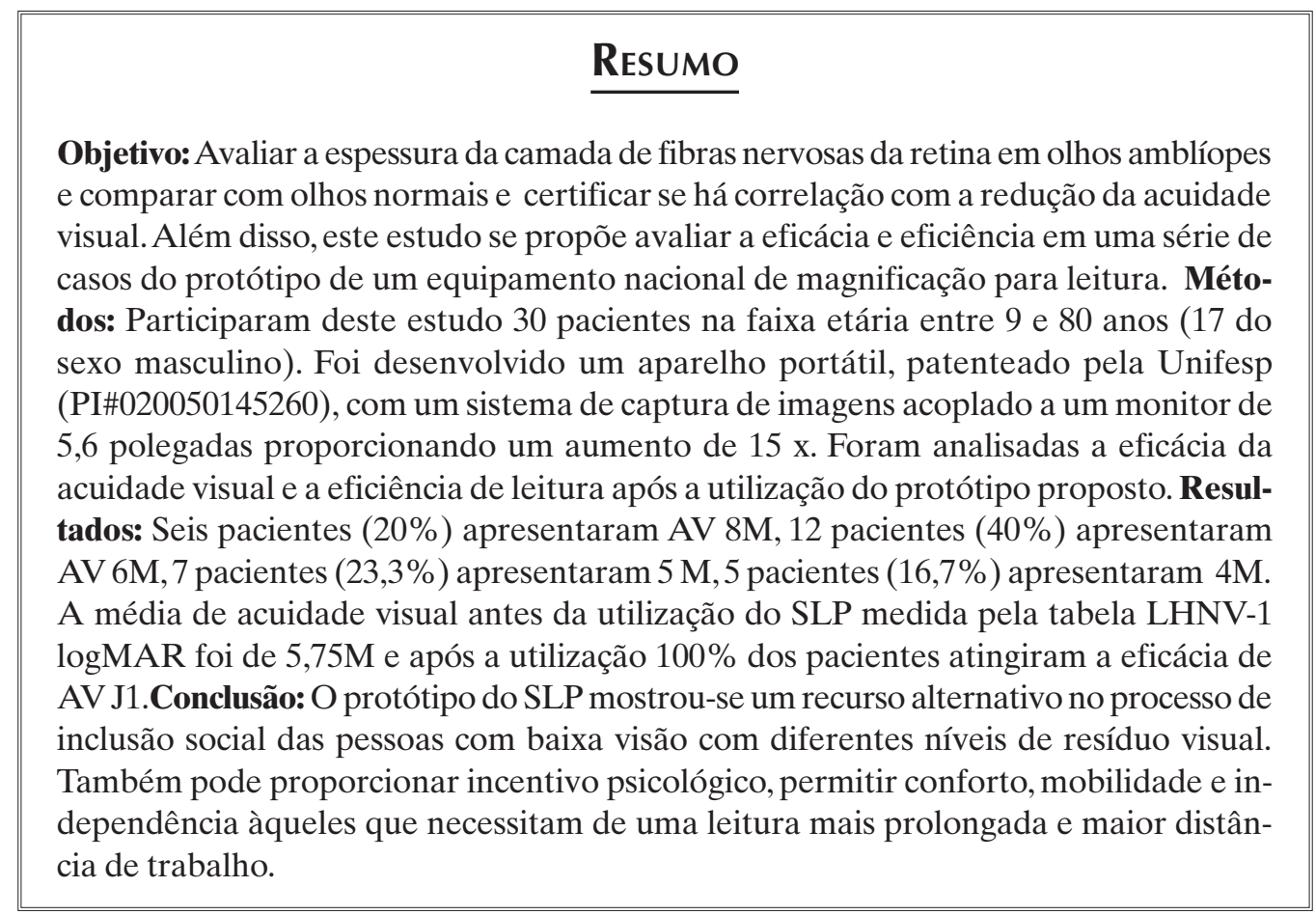

Descritores: Baixa visão/reabilitação; Desenho de equipamento; Engenharia biomédica; Leitura; Qualidade de vida; Brasil

\footnotetext{
${ }^{1}$ Residente do terceiro ano da Disciplina de Oftalmologia na Faculdade de Medicina ABC - FMABC - Santo André (SP), Brasil; ${ }^{2}$ Chefe do setor de Neuroftalmologia na Faculdade de Medicina ABC - FMABC - Santo André (SP), Brasil;

${ }^{3}$ Estagiário do segundo ano da Disciplina de Neuroftalmologia na Faculdade de Medicina ABC - FMABC - Santo André (SP), Brasil;

${ }^{4}$ Tecnóloga da Disciplina de Oftalmologia na Faculdade de Medicina ABC - FMABC - Santo André (SP), Brasil;

${ }^{5}$ Professor Titular, Chefe da Disciplina de Oftalmologia na Faculdade de Medicina ABC - FMABC - Santo André (SP), Brasil.
}

Instituto de Olhos da Disciplina de Oftalmologia da Faculdade de Medicina do ABC - FMABC - Santo André (SP), Brasil.

Recebido para publicação em: 18/8/2009 - Aceito para publicação em 18/10/2009 


\section{INTRODUÇÃO}

A ambliopia é a deficiência visual de desenvolvimento mais comum em humanos, afetando cerca de $2 \%$ da população adulta ${ }^{(1)}$. Em crianças tem uma incidência de $0,5-3,5 \%$ em idade pré-escolar e escolar. Resulta em uma privação na exposição do sistema visual aos estímulos ambientais durante o seu período crítico de desenvolvimento, momento em que a conexão entre as células retinianas e as do córtex cerebral estão em desenvolvimento e maturação ${ }^{(2)}$. Está frequentemente associada a estrabismos e anisometropias, e mais raramente, a opacidades congênitas de meios e ptose ${ }^{(1)}$.

Apesar da extensa documentação acerca das deficiências visuais em amblíopes humanos, os mecanismos responsáveis por estes defeitos ainda permanecem obscuros. Hubel e Wiesel realizaram estudos experimentais pioneiros sobre as possíveis origens neurais da ambliopia, em que observaram alterações corticais importantes em animais submetidos a períodos de privação visual ${ }^{(3)}$. Estes estudos sugerem que o local principal de disfunção neural na ambliopia seja a área V1 do córtex visual.

$\mathrm{O}$ efeito deletério da ambliopia em células do corpo geniculado lateral tem sido bem estabelecido através de estudos histológicos quantitativos em diversas espécies de animais e também em humanos ${ }^{(4)}$. Estas alterações anterógradas, observadas na via visual de pacientes com ambliopia, teriam origem devido a um processo de degeneração secundária transneuronal ou transsinaptica, muito comum em várias regiões cerebrais acometidas por danos funcionais, principalmente durante o período de desenvolvimento ${ }^{(5)}$.

O estudo da camada de fibras nervosas da retina nessas condições é ainda pouco investigado e os trabalhos que descrevem alterações retinianas em olhos amblíopes, com investigação não invasiva, permanecem inconclusivos e controversos ${ }^{(6)}$.

A tomografia de coerência óptica (OCT) é uma técnica recente de exame, capaz de realizar cortes seccionais de tecidos biológicos ${ }^{(7)}$.

Seu princípio assemelha-se muito ao da ecografia modo B, porém ao invés de utilizar ondas sonoras faz uso da luz para obtenção das imagens, o que aumenta muito a sua resolução espacial ${ }^{(8)}$.

Na tomografia de coerência óptica um feixe luminoso é transmitido ao olho. Como a velocidade da luzé infinitamente mais rápida que a do som, ao ser refletida ela retorna à sonda em um tempo da ordem de $10-15$ segundos (femto) - valor que ultrapassa a capacidade de processamento do computador. Desta forma é necessária a utilização de um artifício óptico conhecido como interferômetro de Michelson para a análise deste feixe luminoso.

O interferômetro de Michelson possui um diodo superluminescente que produz feixe de luz de baixa coerência cujo comprimento de onda situa-se próximo da faixa do infravermelho $(820 \mathrm{~nm})$. Este feixe de luz é dividido em dois segmentos, um que vai ao olho e um segundo que é enviado a um espelho de referência cuja distância da fonte luminosa é conhecida ${ }^{(9)}$.

Ao se combinar a luz refletida pelas estruturas oculares mais a luz que retorna do espelho de referência ocorre um processo físico conhecido como interferência. Um fotodetector capta essa interferência e mede as distâncias entre as estruturas oculares, bem como a sua refletividade na resolução espacial da ordem de 10 micrômetros.

O processo é repetido de 128 a 768 vezes ao longo de uma linha, formando a imagem tomográfica. Esta imagem é colorizada pelo computador baseando-se na refletividade da luz captada. Existe boa correlação entre as informações obtidas pela OCT e os aspectos histológicos da retina. Este exame vizualiza as camadas de células da retina com uma resolucão de 10 a $17 \mu \mathrm{m}$ e vem sendo muito usado em casos de retinopatia diabética, glaucoma e degeneração macular. Atualmente tem se tentado estender o uso dessa mesma tecnologia com a finalidade de avaliar as mudanças ocorridas em neuropatias ópticas crônicas e detectar mudanças permanentes das fibras nervosas da retina, para que novas intervenções neuroprotetoras sejam estabelecidas futuramente ${ }^{(10)}$.

\section{Métodos}

O presente estudo foi aprovado pelo comitê de ética em pesquisa sob o protocolo 294-2007.

Foram avaliados 13 pacientes com diagnóstico de ambliopia anisotrópica ou estrabísmica, encaminhados do Ambulatório de Oftalmologia da Faculdade de Medicina do ABC. Os voluntários foram de ambos os sexos, com faixa etária acima de 18 anos, apresentando ambliopia profunda, sendo que no melhor olho a acuidade visual é de $0.0 \log$ MAR ou melhor.

Todos os pacientes foram submetidos a um exame oftalmológico e medida da acuidade visual monocular com a tabela ETDRS e exame de tomografia de coerência óptica em ambos os olhos, sob midríase medicamentosa, após a assinatura do termo de consentimento livre e esclarecido.

Foram excluídos pacientes com outras patologias oftalmológicas ou neurológicas, história de tabagismo e alcoolismo, cirurgias oftalmológicas prévias (exceto aque- 
las para correção de estrabismo) e patologias sistêmicas com repercussão oftalmológica ou neurológica.

\section{Protocolo de Testagem do OCT}

Para avaliar a espessura da camada de fibras nervosas da retina foi utilizado o Humphrey-Zeiss Stratus (OCT3) (Carl Zeiss-Humphrey-Meditec ${ }^{\circledR}$, Dublin, Califórnia, USA) com software 4.0.3.1. Foram utilizadas as estratégias fast RNFL e medidas da espessura e volume macular. O olho com melhor acuidade visual foi testado primeiro para facilitar o entendimento do exame pelo paciente. Foi realizada uma segunda avaliação logo após a primeira para confirmar as medidas.

O exame foi realizado sob midríase medicamentosa, com colírio de tropicamida a $1 \%$ e fenilefrina a $10 \%$.

\section{Análise estatística}

O método estatístico empregado foi o de amostras pareadas de Wilcoxon. Este método corrige as alterações individuais tais como: diferença de espessura, refletividade e topografia das diferentes camadas da retina e da papila óptica, pois compara as amostras pareadas, neste caso, o olho direito com o esquerdo.

A acuidade visual foi apresentada em $\log M a r$ e comparada entre o olho direito com o esquerdo do mesmo paciente, usando este mesmo cálculo estatístico.

\section{Resultados}

\section{Dados utilizados}

Teste de Wilcoxon - Acuidade visual (AV): olho: Direito (_d) x Esquerdo (_e)

Test Statistics ${ }^{b}$

\begin{tabular}{cccc}
\hline & N & $\begin{array}{r}\text { Mean } \\
\text { Rank }\end{array}$ & $\begin{array}{c}\text { Sum of } \\
\text { Ranks }\end{array}$ \\
\hline AV_e - AV_d Negative Ranks & 3 & 5,50 & 16,50 \\
Positive Ranks & 4 & 2,88 & 11,50 \\
Total & 7 & & \\
\hline
\end{tabular}

\begin{tabular}{lr}
\hline & AV_e - AV_d \\
\hline Z &,$- 424^{\mathrm{a}}$ \\
Asymp. Sig. (2-tailed) &, 671 \\
$\mathrm{p}=$ & \\
\hline
\end{tabular}

a. Based on positive ranks

b. Wilcoxon Signed Ranks Test

\begin{tabular}{ccccc}
\multicolumn{5}{c}{ Descritiva } \\
\hline & N & Média & Minímo & Máximo \\
\hline AV_d & 12 & 60 & 20 & 200 \\
AV_e & 8 & 70 & 20 & 150
\end{tabular}

Obs: Estatisticamente não significante para acuidade visual $(\mathrm{AV}) ; \mathrm{z}=0,424 ; \mathrm{p}=0,671 ;$ média $\mathrm{OD}=59$ e $\mathrm{OE}=7$ Teste de Wilcoxon - espessura papilar (nasal; temporal; superior; inferior) -

Olho: Normal (_d) x Amblíope (_e)

Ranks

\begin{tabular}{llrcc}
\hline & & N & Mean Rank & Sum of Ranks \\
\hline \multirow{2}{*}{ Nasal_e - Nasal_d } & Negative Ranks & 9 & 7,50 & 67,50 \\
& Positive Ranks & 4 & 5,88 & 23,50 \\
\multirow{2}{*}{ Temporal_e - Temporal_d } & Total & 13 & & \\
& Negative Ranks & 5 & 9,30 & 46,50 \\
& Positive Ranks & 7 & 4,50 & 31,50 \\
Superior_e - Superior_d & Total & 13 & & 58,00 \\
& Negative Ranks & 7 & 8,29 & 33,00 \\
\multirow{2}{*}{ Inferior_e - Inferior_d } & Positive Ranks & 6 & 5,50 & 52,00 \\
& Total & 13 & & 39,00 \\
& Negative Ranks & 8 & 6,50 & \\
& Positive Ranks & 5 & 7,80 & \\
\hline
\end{tabular}


Test Statistics ${ }^{b}$

\begin{tabular}{lcccc}
\hline & $\begin{array}{c}\text { Nasal_e - } \\
\text { Nasal_d }\end{array}$ & $\begin{array}{c}\text { Temporal_e - } \\
\text { Tempora__d }\end{array}$ & $\begin{array}{c}\text { Superior_e - } \\
\text { Superior_d }\end{array}$ & $\begin{array}{c}\text { Inferior_e - } \\
\text { Inferior_d }\end{array}$ \\
\hline $\begin{array}{l}\text { Z } \\
\text { Asymp. Sig. (2 tailed) } \\
\text { p= }\end{array}$ & $-1,540^{\mathrm{a}}$ &,$- 589^{\mathrm{a}}$ &,$- 875^{\mathrm{a}}$ &,$- 455^{\mathrm{a}}$ \\
\hline
\end{tabular}

a. Based on positive ranks.

b. Wilcoxon Signed Ranks Test

\begin{tabular}{lcccc}
\multicolumn{5}{c}{ Descritiva } \\
\hline & N & Média & Mínimo & Máximo \\
\hline Nasal_d & 13 & 91,5 & 60 & 135 \\
Temporal_d & 13 & 70,2 & 56 & 82 \\
Superior_d & 13 & 123,5 & 96 & 147 \\
Inferior_d & 13 & 131,8 & 112 & 147 \\
Nasal_e & 13 & 82,3 & 58 & 117 \\
Temporal_e & 13 & 68,2 & 47 & 86 \\
Superior_e & 13 & 121,2 & 96 & 142 \\
Inferior_e & 13 & 130,6 & 117 & 155 \\
\hline
\end{tabular}

Obs: Estatisticamente não significante para as diferentes espessura papilares Nasal: $\mathrm{z}=1,540 ; \mathrm{p}=0,124$; média olho normal $=91,5$ e olho amblíope $=82,3$;

Temporal: $\mathrm{z}=0,589 ; \mathrm{p}=0,556$; média olho normal $=70,2$ e olho amblíope $=68,2$; Superior: $\mathrm{z}=0,875 ; \mathrm{p}=0,381$; média olho normal $=123,5$ e olho amblíope $=121,2$; Inferior: $\mathrm{z}=0,455 ; \mathrm{p}=0,649$; média olho normal $=131,8$ e olho amblíope $=130,6$; Teste de Wilcoxon - Espessura macular - olho: Direito (_d) x Esquerdo (_e)

Ranks

\begin{tabular}{lllll}
\hline & \multicolumn{3}{c}{$\begin{array}{c}\text { Sum } \\
\text { Mean of } \\
\end{array}$} & N Rank Ranks \\
\hline Macular_e - Macular_d & Negative Ranks & 4 & 4,75 & 19,00 \\
& Positive Ranks & 8 & 7,38 & 59,00 \\
& Total & 13 & & \\
\hline
\end{tabular}

Test Statistics ${ }^{b}$

\begin{tabular}{lc}
\hline & $\begin{array}{c}\text { Macular_e - } \\
\text { Macular_d }\end{array}$ \\
\hline $\mathrm{Z}$ & $-1,570 \mathrm{a}$ \\
Asymp. Sig. (2-tailed) &, 117 \\
\hline a. Based on negative ranks & \\
b. Wilcoxon Signed Ranks Test &
\end{tabular}

\section{Descritiva}

\begin{tabular}{lcccc}
\hline & N & Média & Minímo & Máximo \\
\hline Macular_d & 13 & 153,2 & 128 & 201 \\
Macular_e & 13 & 172,7 & 128 & 226 \\
\hline
\end{tabular}

Obs: Estatisticamente não significante para a espessura macular;

$\mathrm{z}=1,570 ; \mathrm{p}=0,17 ;$ média olho normal $=153,2$ e olho amblíope $=172,7$

\section{Testes de Wilcoxon}

- A comparação entre as medidas do olho esquerdo e direito, aplicando-se o teste de Wilcoxon para as 6 variáveis consideradas no estudo, não difere estatisticamente pois $\mathrm{p}>0,05(\mathrm{p}>5 \%)$, em todos os casos.

\section{DiscussÃo}

Os mecanismos causadores da ambliopia são: estimulação visual inadequada na fóvea durante a infância, deprivação visual, interação binocular 
anormal e incongruência das informações visuais recebidas pelos dois olhos ${ }^{(10)}$. Essas condições causam anormalidades comportamentais e neurofisiológicas no córtex estriado e pré-estriado e redução do tamanho das células do corpo geniculado lateral $^{(2)}$. Um estudo histológico de pacientes com ambliopia anisometrópica revelou um decréscimo do tamanho das células nas camadas parvocelulares inervadas pelas fibras provindas do olho amblíope ${ }^{(11)}$

Todas essas alterações descritas acima já foram comprovadas, porém o envolvimento retiniano em olho amblíopes é controverso ${ }^{(12)}$. Muitos experimentos têm demonstrado que células ganglionares retinianas podem sofrer modificações com privação da luz desde o nascimento,incluindo perda celular, diminuição do volume nucleolar do citoplasma nas células ganglionares e afinamento da camada plexiforme interna em ratos e $\operatorname{gatos}^{(13) \text {. }}$

Arden and Wooding relataram que eletroretinogramas realizados em humanos com vários tipos de ambliopia foram significativamente alterados. Esses resultados sugerem que em humanos, ambliopia provavelmente associa-se com mudanças nas funções retinianas, as quais parecem ser préganglionares $^{(14)}$.

Em um estudo, usando O.C.T, Yen et al. revelou um afinamento nas camadas de fibras nervosas retinianas em olhos com ambliopia refracional, com uma considerável significância estatística, quando comparados com olhos amblíopes do mesmo paciente ${ }^{(15)}$.

No presente estudo, a camada de fibras nervosas peripapilares e a espessura macular foram medidas através do O.C.T e os resultados não foram estatisticamente significantes.

A média da espessura foveal em nosso estudo foi: $z=1,570 ; \mathrm{p}=0,17$; média olho normal $=153,2$ e olho amblíope $=172,7$.

Já a média da espessura peripapilar foi:

Nasal: $\mathrm{z}=1,540 ; \mathrm{p}=0,124$; média olho normal $=91,5 \mathrm{e}$ olho amblíope $=82,3$;

Temporal: $\mathrm{z}=0,589 ; \mathrm{p}=0,556 ;$ média olho normal $=70,2$ e olho amblíope $=68,2$;

Superior: $\mathrm{z}=0,875 ; \mathrm{p}=0,381 ;$ média olho normal $=123,5$ e olho amblíope $=121,2$;

Inferior: $\mathrm{z}=0,455 ; \mathrm{p}=0,649$; média olho normal $=131,8$ e olho amblíope $=130,6$.

\section{Conclusão}

Embora a amostra utilizada nesse estudo tenha sido pequena, os resultados sugeriram que o processo de ambliopia provavelmente não tem um significante efeito na mácula e nas fibras nervosas peripa.

\section{Abstract}

Objective: To compare the thickness of the retinal nerve fiber layer (RNFL) and the macular thickness of the amblyopic eye with those of the non-amblyopic eye in patients with unilateral amblyopia using optical coherence tomography (OCT). Methods: OCT was performed for 13 patients with unilateral amblyopia who had no neurologic disease. Nine male and four female patients, whose ages ranged from 23 to 63 years, were enrolled in the study. The RNFL thickness average analysis program was used to evaluate mean superior, inferior, temporal, and nasal thickness. The data for all clock quadrants (12 values averaged) were identified as the overall RNFL. The retinal thickness analysis program was used to evaluate macular scans. Data were compared using the Man $n$-Whitney U test. Results: The mean age ( standard deviation) was 35,43 years. There were 13 eyes with amblyopia; this group had visual acuity 0,1 $\log M A R$ or better in the best eye. OCT parameters including the RNFL thickness in all quadrants, overall RNFL thickness and macular thickness showed no significant differences between the two groups $(p>0,5)$. Conclusion: Assessment of RNFL thickness and macular thickness with OCT revealed no difference between the two eyes of patients with unilateral amblyopia.

Keywords: Nerve fibers/pathology; Macula lutea/ anatomy \& histology; Amblyopia/complications; Retinal ganglion cells/pathology; Retina/pathology; Tomography, optical coherence.

\section{ReferênCIAS}

1. Anderson SJ, Holliday IE, Harding GF. Assessment of cortical dysfunction in human strabismic amblyopia using magnetoencephalography (MEG). Vision Res. 1999;39(9):1723-38

2. von Noorden GK. . Mechanism of amblyopia.. Doc Ophthalmol. 1977; 34: 93.

3. Wiesel TN, hubel DH. Effects of visual deprivation on morphology and hysiology of cells in the cats lateral geniculate body. J Neurophysiol. 1963;26:978-93. 
4. Asch E, Swift H, Riesen AH, Chow KL. Altered structure and composition of retinal cells in darkreared mammals. Exp Cell Res. 1961;25:348-63

5. Miller N, Newman N, Biousse V, Kerrison J. Walsh \& Hoyt's clinical neuro-ophthalmology: The essentials. 5th ed. New York: Lippincott Williams \& Wilkins, 1999.

6. Frohman E, Costello F, Zivadinov R, Stuve O, Conger A, Winslow H, Trip A,Frohman T, Balcer L. Optical coherence tomography in multiple sclerosis. Lancet Neurol. 2006;5(10):853-63. Review.

7. Chauhan DS, Marshall J. The interpretation of optical coherence tomography images of the retina. Invest Ophthalmol Vis Sci. $1999 ; 40(10): 2332-42$.

8. Hee MR, Izatt JA, Swanson EA, Huang D, Schuman JS, Lin CP, Puliafito CA, Fujimoto JG. Optical coherence tomography of the human retina. Arch Ophthalmol.1995;113(3):325-32.

9. Trobe JD, Glaser JS, Cassady JC. Optic atrophy. Differential diagnosis by fundus observation alone. Arch Ophthalmol. 1980;98(6):1040-5.

10. Friedman Z, Neumann E, Hyams SW, Peleg B. Ophthalmic screening of 38,000 children, age 1 to $21 / 2$ years, in child welfare clinics. J Pediatr Ophthalmol Strabismus. 1980; 17(4):261-7.
11. Sherman SM, Wilson JR. Behavioral and morphological evidence for binocular competition in the postnatal development of the dog's visual system. J Comp Neurol. 1975;161(2):183-95

12. Vereecken E, Feron A, Evens L. [The importance of early detection of trabismus and amblyopia]. Bull Soc Belge Ophtalmol. 1966;143:729-39. French.

13. Wendell-Smith CP. Effect of light deprivation on the postnatal development of the optic nerve. Nature. 1964;204:707.

14. Arden GB, Wooding SL. Pattern ERG in amblyopia. Invest Ophthalmol Vis Sci. 1985;26(1):88-96.

15. Yen MY, Cheng CY, Wang AG. Retinal nerve fiber layer thickness in unilateral amblyopia. Invest Ophthalmol Vis Sci. 2004;45(7):2224-30.
Endereço para Correspondência
Juliana Mitre
Rua Casa Forte, $n^{\circ} 347$
São Paulo (SP), Brasil
Tel: (11) 89099025
E- mail: julianamitre@yahoo.com.br 